\title{
Lung Development in the Chick Embryo. I. Phosphatidylcholine Synthesis in the Developing Chick Lung
}

\author{
SUZANNE K. COMPTON ${ }^{(24)}$ AND GERALD C. GOERINGER ${ }^{(26)}$ \\ Department of Anatomy, Georgetown University Schools of Medicine and Dentistry, Washington, D. C., USA
}

\begin{abstract}
Summary
We investigated the relative contribution of the two pathways of phosphatidylcholine (PC) synthesis in lungs from chick embryos of 12 to 20 days of incubation as well as from hatched chicks and adult chickens. Lung "blocks" were incubated for $60 \mathrm{~min}$ in vitro in the presence of $\left[{ }^{3} \mathrm{H} \mid\right.$ methionine and $\left[{ }^{14} \mathrm{C} \mid\right.$ choline. $\mathrm{PC}$ was separated by thin-layer chromatography, and the amount of labeled product was determined by liquid scintillation counting. PC synthesis (expressed as pmoles PC per $\mathrm{mg}$ protein per $60 \mathrm{~min}$ ) by the choline pathway declined from the 12-day value of 40 to a minimum of 8 at day 16 and then rose gradually to a peak of 74 on day 1 posthatching. PC synthesis via the phosphatidylethanolamine pathway also declined from day $12(54)$ to day $16(13)$ but peaked on day 19 (65) and again on day 1 posthatching (54). Synthetic activities of the two pathways differed significantly $(P \leq 0.05)$ only on days 14, 18, and 19 of incubation. Unlike the mammalian lung, neither pathway seems to predominate throughout incubation or after hatching. The increase in PC synthesis towards the end of incubation correlates with the time of appearance of lamellar bodies in the lung epithelial cells and of surfactant in the airways.
\end{abstract}

\section{Speculation}

Because normal morphologic and biochemical development of the chick lung resembles that occurring in the mammalian lung in many respects, conclusions drawn from the study of avian lungs may well be germane to an appreciation of mammalian lung differentiation in general. Information concerning regulation of the biosynthetic pathways for phosphatidylcholine in the avian embryo may provide some clues for improved clinical treatment of certain pulmonary disorders, including respiratory distress syndrome of the newborn.

Until recently, the question of whether or not the avian lung possessed surfactant was a matter of controversy (17). Now this material has been demonstrated lining the parabronchial, atrial, and air capillary membranes in the lungs of several avian species $(18,19)$. Cells resembling mammalian types I and II pneumocytes have been described by several investigators in the adult and embryonic chick lung (14, 21).

The major component of avian pulmonary surfactant is phosphatidylcholine (PC) $(16,21)$. Two pathways are involved in the de novo synthesis of PC: the choline incorporation pathway (I) and the phosphatidylethanolamine (PE) methylation pathway (II). Fetal and adult lungs of all mammalian species examined to date have been shown to possess the enzymes necessary for each pathway $(1,6,7)$. The choline incorporation pathway has been identified as the major route for PC synthesis in the adult and late fetal mammalian lung (5). No studies have been published on the pathways for PC synthesis in the embryonic, newly hatched, or adult chicken lung.

We investigated the production of PC in blocks of lungs from chicks of different ages. The blocks were incubated in the presence of radioactive precursors specific to the two pathways for PC synthesis. This provided us with a measure of the relative importance of each pathway in the embryonic and young chick lung.

\section{MATERIALS AND METHODS}

\section{EGGS}

Fertile White Leghorn hens' eggs (Truslow Farms, Chestertown, MD) from 12 to 20 days of incubation were utilized. The developmental age of the chick embryo was determined by (1) days of incubation and (2) staging of the embryo using the criteria established by Hamburger and Hamilton (13). Young chicks were obtained from the same source.

\section{ISOTOPE INCORPORATION PROCEDURE}

Lungs from chick embryos, newly hatched chicks, and 16- and 23-day (posthatching) chicks were rapidly removed and placed in ice-cold saline until used. Four $\mathrm{ml}$ of Krebs-Ringer bicarbonate solution, $\mathrm{pH} 7.6$, containing dextrose $(2 \mathrm{mg} / \mathrm{ml})$ were added to 25-ml Erlenmeyer flasks. Lungs were chopped with razor blades into blocks of approximately $1 \mathrm{~mm}^{3}$ and $300 \mathrm{mg}$ of this tissue were placed in each flask. Triplicate samples from each age period were prepared. The flasks were preincubated for $10 \mathrm{~min}$ at $37^{\circ} \mathrm{C}$ in a rotary shaking water bath at $100 \mathrm{rpm}$ under a steady flow of $95 \%$ $\mathrm{O}_{2}: 5 \% \mathrm{CO}_{2}$. To each flask was added $2 \mu \mathrm{Ci}$ of $\left[\right.$ methyl $\left.-{ }^{14} \mathrm{C}\right]$ choline chloride (Amersham Searle; specific activity $=52 \mu \mathrm{Ci} / \mu$ mole) and $2 \mu \mathrm{Ci}$ of $\mathrm{L}-\left[\right.$ methyl $\left.-{ }^{3} \mathrm{H}\right]$ methionine (Amersham Searle; specific activity $=52 \mu \mathrm{Ci} / \mu$ mole $)$.

Duration of incubation was determined in a preliminary experiment in which lung tissue from 19-day embryos was incubated for 40,80 , and $120 \mathrm{~min}$. Incorporation was still linear after 120 min. Subsequently, lung samples were incubated for $60 \mathrm{~min}$. Aliquots $(0.05 \mathrm{ml})$ of the medium taken both before and after incubation were counted in Aquasol in a Searle liquid scintillation counter. After incubation, samples were placed on ice until lipids could be extracted.

\section{EXTRACTION AND QUANTITATION OF LIPIDS}

Each lung sample was homogenized in a Dounce homogenizer at $4^{\circ} \mathrm{C}$. This homogenate was used for both lipid and protein determinations. Total lipids were extracted from an aliquot of the lung homogenate according to Folch et al. (8). An aliquot from the aqueous phase of each Folch extract was taken for liquid scintillation counting to determine the amount of labeled precursors and intermediates present in lung tissue after incubation. Cold precursor "pools" of choline and methionine were not measured in any of the lung extracts. The chloroform phase was evaporated under $\mathrm{N}_{2}$ and redissolved in $0.3 \mathrm{ml}$ of hexane. Each sample was spotted onto silicic acid chromatographic plates (Eastman Kodak No. 6061). Standards of complex (Applied Science 
Lab TLC Standard No. 21994) and neutral lipids (Applied Science Lab TLC Standard No. 21999) were used for identification of individual lipid fractions. The plates were run in the solvent system chloroform:methanol:water (168:72:24). After the run, the plate was air-dried and developed in iodine fumes for identification of lipids. Each sample was separated into four fractions: origin and lysolecithin, PC, PE, and neutral fats. No attempt was made to identify that portion of the PC fraction which was dipalmitoyl PC. Individual spots were cut out and placed in $4 \mathrm{ml}$ of 4.2\% PPO:POPOP (Liquifluor, New England Nuclear). All samples were counted in a Searle liquid scintillation counter using a program which counts ${ }^{3} \mathrm{H}$ and ${ }^{14} \mathrm{C}$ simultaneously (the window for each isotope was preset and dpm calculated automatically by the counter).

Protein content of each sample was determined according to Lowry et al. (15). The amount of PC synthesized from each labeled precursor was calculated (using specific activity) and expressed as pmoles of $\left[{ }^{14} \mathrm{C}\right] \mathrm{PC}$ or $\left[{ }^{3} \mathrm{H}\right] \mathrm{PC}$ synthesized per mg protein per 60 min. Mean and standard error of the mean were calculated for each time period. The difference between means was tested using the two-tailed Student's $t$ test at the $P \leq 0.05$ level.

\section{RESULTS}

Incorporation of ${ }^{3} \mathrm{H}$-methyl groups from methionine and $\left[{ }^{14} \mathrm{C}\right]$ choline into PC in the 19-day embryonic chick lungs was still linear at $120 \mathrm{~min}$ (Fig. 1). No significant difference was found between ${ }^{3} \mathrm{H}$ - and ${ }^{14} \mathrm{C}$-labeled precursors or intermediates found in the lung tissue at any age studied. At least $75 \%$ of both labels was found in the medium after $60 \mathrm{~min}$ incubation for all time periods.

Synthesis of PC by both pathways is traced in Figure $2 .\left[{ }^{14} \mathrm{C}\right]$ choline incorporation into PC decreased significantly between days 12 and 14 and again between days 14 and 16. Although the successive daily differences in choline incorporation into PC between days 16 through 19 were not significant, the overall rise from days 16 through 19 was. On day 20, two different groups of lungs were examined: those from embryos that had begun to breathe air ("20-day air"- the lung floated) and those from embryos that had not yet begun a significant amount of airbreathing ("20-day non-air"). No significant difference in PC synthesis was found between these two 20-day groups. PC synthesis by pathway I peaked on day 1 after hatching and decreased significantly through day 23 posthatching, the last stage at which samples were assayed. Choline incorporation was not significantly greater right before or after hatching than it was on day 12 of incubation (Fig. 2).

Incorporation of ${ }^{3} \mathrm{H}$-methyl groups from methionine into PC followed a time course showing minima at days 16 and 20 (after air was in the lungs) and again at days 16 and 23 after hatching. Activity peaked at day 19 with a secondary increase on day 1 after hatching. Maximum values in pmoles of PC produced per mg protein per hr were 54 at day 12 and 65 (day 19) and 54 on day 1 posthatching. Minimum values were 13 (day 16), 34 (day 20 air in lungs), and 32 and 34 (days 16 and 23 posthatching).

Values for the PE methylation pathway were higher than for the choline incorporation pathway at all days of incubation examined but were significantly greater only on days 14, 18, and 19 . No significant difference was found between the amounts of PC synthesized by either pathway after hatching.

After chromatographic separation of esterified ${ }^{3} \mathrm{H}$-lipids, 77 to $92 \%$ of the counts was found in the PC fraction, whereas approximately $10 \%$ of the radioactivity either remained at the origin or was incorporated into lysolecithin. The PE fraction contained 3\% of the counts, and $3 \%$ was in neutral fats. Of the counts found in esterified ${ }^{14} \mathrm{C}$-lipids, 77 to $96 \%$ was in the PC fraction. Approximately $13 \%$ of the label remained at the origin or was in the lysolecithin fraction, and less than $1 \%$ was found in either PE or neutral fats.

\section{DISCUSSION}

Relatively little work has been published on the biosynthesis of surfactant in the embryonic or young chicken lung. Adult turkey lung surfactant has the same phospholipid, PC, PE, and protein composition as rabbit lung surfactant (9). Cells which, at the ultrastructural level, resemble type II pneumocytes are found in

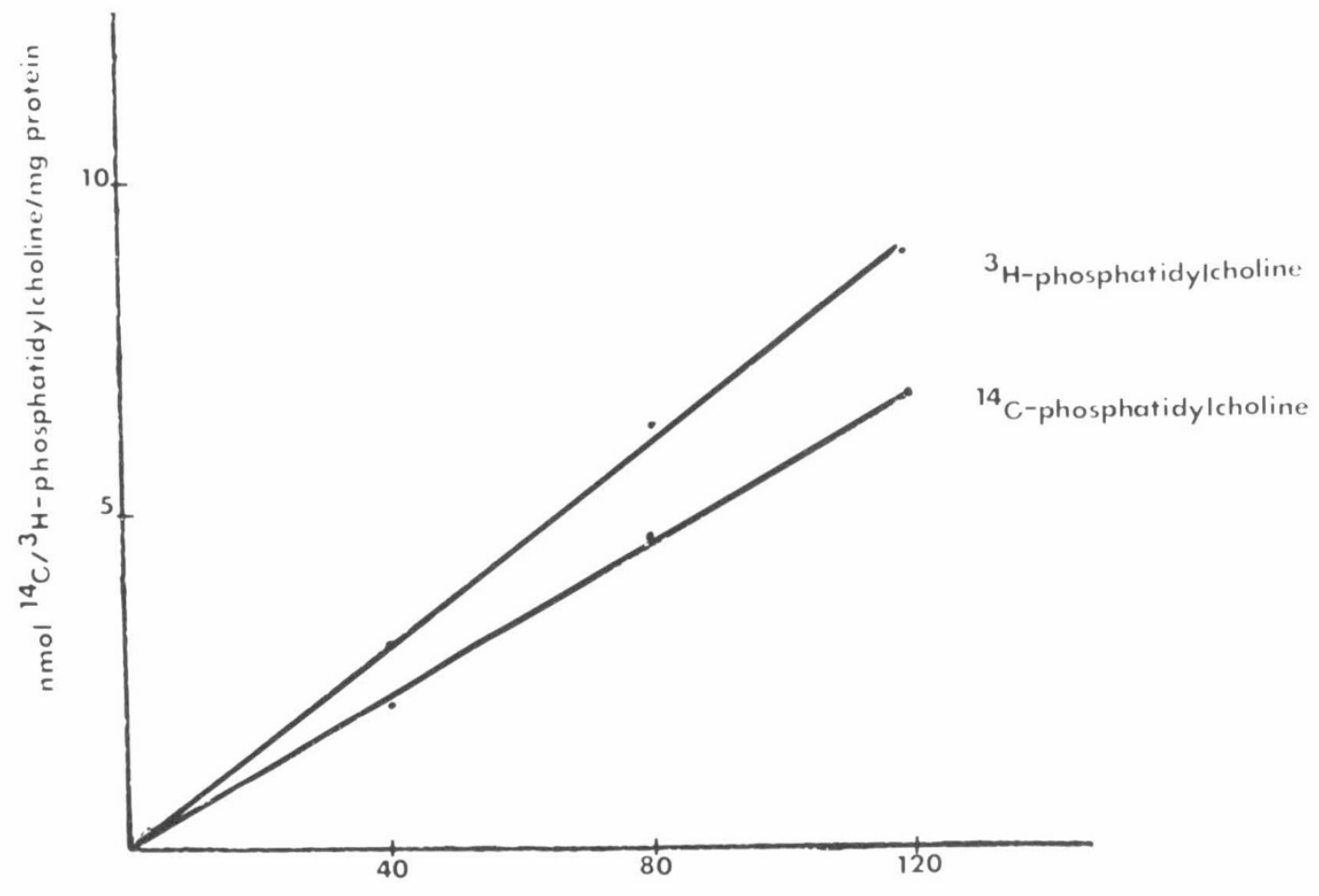

Incubation time in minutes

Fig. 1. Incorporation of ${ }^{3} \mathrm{H}$-methyl groups from methionine and $\left[{ }^{14} \mathrm{C}\right]$ choline into phosphatidylcholine as a function of duration of incubation. 


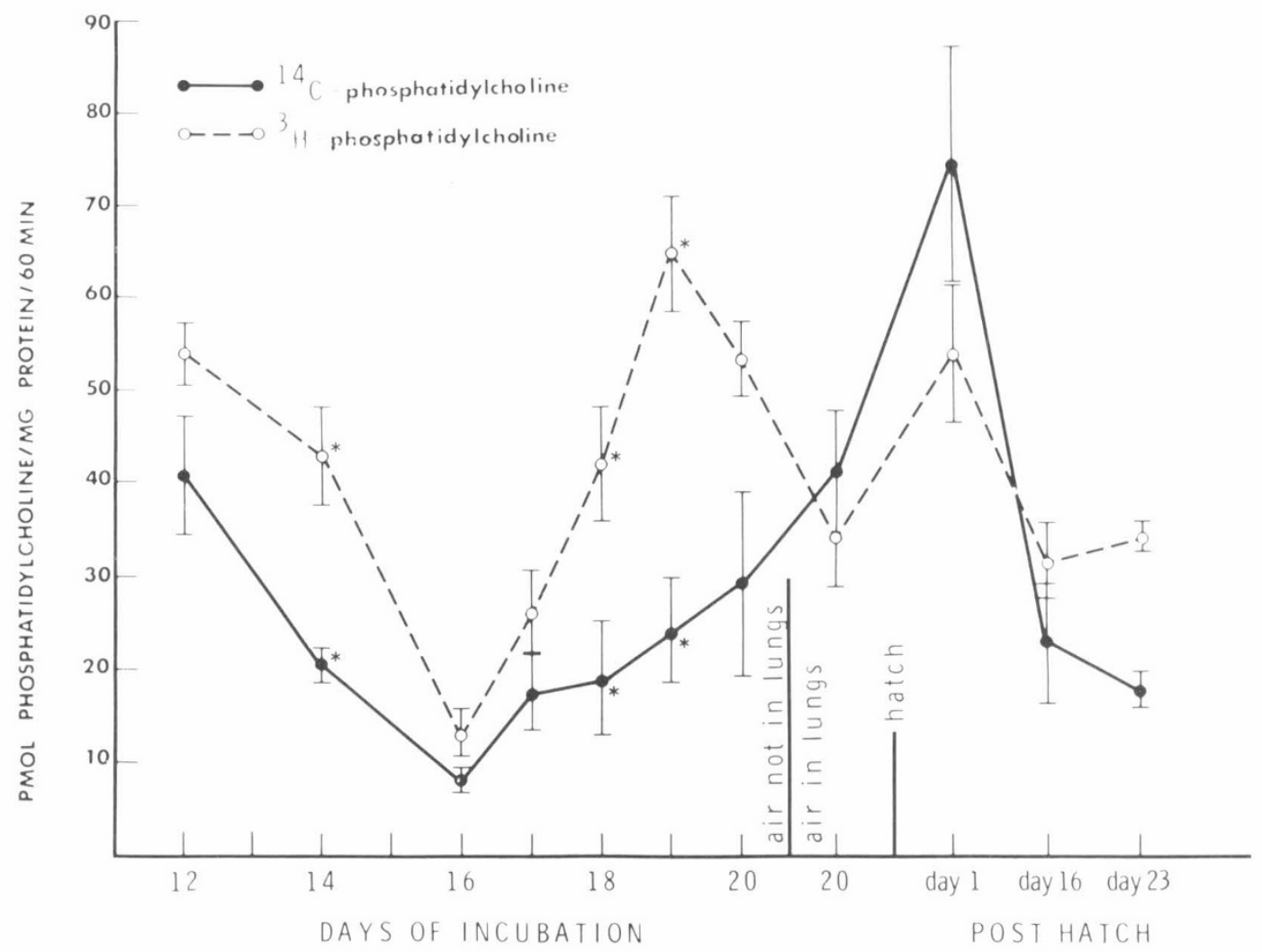

Fig. 2. Incorporation of $\left[{ }^{14} \mathrm{C}\right]$ choline $(-)$ and ${ }^{3} \mathrm{H}$-methyl groups from methionine $(\bigcirc)$ into phosphatidylcholine in lungs of developing chick embryos. Points, mean of three samples. Vertical bars, S.E. Asterisks, days on which the difference between the amount of $\left[{ }^{14} \mathrm{C}\right]$ phosphatidylcholine and $\left[{ }^{3} \mathrm{H}\right]$ phosphatidylcholine synthesized was significant $(P \leq 0.05)$.

the atria of the avian lung and are believed to be the source of this lining material (18).

The purpose of this study was to determine if the two pathways for PC synthesis, the choline incorporation and the PE methylation pathways, were operational in the developing chick lung and if, as in the mammalian lung, the choline incorporation pathway plays the major role in PC synthesis.

We found that neither pathway seemed to predominate throughout most of the period of in ovo development or after hatching. Incorporation of ${ }^{3} \mathrm{H}$-methyl groups from methionine into PC was slightly higher than choline incorporation during the embryonic period but only significantly so on days 14,18 , and 19 of incubation. However, because we did not take into account the size of the "cold" precursor pools in the lung tissue, our in vitro incorporation studies did not measure absolute synthetic rates by both pathways. No studies have reported the amount of choline, ethanolamine, or methionine in the developing chick lung. Gail and Farrell (10) reported that, in the adult rat lung, choline, ethanolamine, and methionine were present in equivalent amounts. Choline content of the fetal rat lung late in gestation was the same as in the adult, however, ethanolamine and methionine levels were higher. If the same holds true for the embryonic chick lung, the larger pool of cold methionine in the tissue would make pathway II appear less active in comparison with pathway I than it is in reality.

Alterations in the rate of synthesis of PC may reflect alterations in the rate of synthesis of all cellular membranes, of which PC is a major component (22). The high incorporation of precursors into PC early in incubation, at days 12 and 14 , is probably due to the active growth of the bronchial tubules and the blood capillaries at these stages.

On day 16 of incubation, lamellar bodies begin to appear in the atrial cells of the chick lung. Marin et al. (16) reported an increase in total phospholipid content of the embryonic chick lung from day 14 to a peak on day 19 followed by a decline in total phospholipid content just before hatching. Surfactant appears in the airways of the chick lung on day 20 of incubation just before hatching (21). Therefore, the rise in incorporation we described after day 16 may be due in part to the onset of synthesis of dipalmitoyl phosphatidylcholine (DPPC). Compared with membrane PC molecules, DPPC, at least in mammals, has a much higher turnover rate. Therefore, incorporation rates such as described here would tend to selectively reflect the surface-active PC production (7). However, because no attempt was made to identify that portion of the PC fraction which was surfactant DPPC, we cannot say if one or both pathways are responsible for the synthesis of surfactant DPPC in the chick lung or if perhaps one pathway succeeds the other.

We found an increase in incorporation of precursors into PC just before hatching, peaking on day 19 of incubation. We have also reported increases in choline kinase and ethanolamine kinase activities on this same day in the embryonic chick lung (3, 4). A similar increase in lung PC levels has been reported in the primate lung just before birth (5). The reservoir theory, proposed by Brumley et al. (2) and others maintains that the fetus must establish high lung PC levels before delivery to withstand any stresses of the immediate postnatal period which might result in a transient decrease in PC synthesis (5). The embryonic chick lung begins to take over the respiratory functions of the chorioallantoic membrane by day 19 of incubation. A "reservoir" of PC sufficient to carry the embryo through hatching might be essential during this transitional period. The increase in PC synthesis by both pathways found on the first day after hatching may serve to compensate for an increased loss of surfactant during this hatching process when air-lung interaction commences.

Pituitary regulation of the adrenal gland begins at days 13 to 15 in the chick embryo and significant increases in plasma corticosterone occur on days 16, 18, and 20 (23). These increases occur just before the increases in PC synthesis which we observed on days 17 and 19 of incubation and on day one after hatching. Plasma corticosterone levels in the normal chick are highest at hatching and decrease within 1 wk. We found PC synthesis by 
both pathways was very high right after hatching and decreased over the following 16 days.

According to Goldfine (12), the biosynthesis of PC was a late evolutionary development compared to the appearance of other phospholipids, and the PE methylation pathway was the first to evolve. There may be a selective advantage to the choline incorporation pathway in the mammalian lung (which may not hold true in the chick lung). One factor could be the greater availability of choline in mammalian tissues. Sim and Gwee (20) reported higher levels of tissue choline in the adult rabbit and guinea pig, especially in the lung, than in the adult chicken, tortoise, or toad. Higher levels of endogenous tissue choline may thus be responsible for the importance of the choline incorporation pathway in the mammalian lung.

It has been suggested that functioning of the PE methylation pathway may be more susceptible to such acute physical and biochemical stresses of birth and the immediate postnatal period as acidosis and hypothermia (11). These conditions may not be as potentially stressful in the chick embryo at hatching where the hatching period extends over a period of $24 \mathrm{hr}$ and more.

\section{CONCLUSION}

Contrary to the situation in the developing mammal, where the choline incorporation pathway is dominant, in the chick both pathways are operative with neither predominating during most of in ovo development or after hatching. Peaks of incorporation occurred on day 19 of incubation and on the first day after hatching, just after the levels of corticosterone circulating in the plasma have been reported to increase.

\section{REFERENCES AND NOTES}

1. Akino, T., Abe, M., and Arai, T.: Studies on the biosynthetic pathways of molecular species of lecithin by rat lung slices. Biochem. Biophys. Acta, 248: 274 (1971).

2. Brumley, G., Hodson, A., and Avery, M. E.: Lung phospholipids and surface tension correlations in infants with and without hyaline membrane disease and in adults. Pediatrics, 40: 13 (1967)

3. Compton, S., and Goeringer, G.: Choline kinase activity in the lungs of normal and hypophysectomized chick embryos. Anat. Rec., 193: 509 (1979).

4. Compton, S., and Goeringer, G.: Choline kinase and ethanolamine kinase activities in the developing chick lung. Am. Rev. Respir. Dis., I2I: 328 (1980).

5. Epstein, M. F., and Farrell, P. M.: The choline incorporation pathway: primary mechanism for de novo lecithin synthesis in fetal primate lung. Pediatr. Res., 9. 658 (1975).

6. Farrell, P. M., and Avery, M. E.: Hyaline membrane disease. In: J. F. Murray: Lung Disease - State of Art. pp. 1-32 (American Lung Association, New York, 1976)
7. Farrell, P. M., and Hamosh, M.: The biochemistry of fetal lung development. Clin. Perinatol., 5: 197 (1978).

8. Folch, S., Less, M., and Sloane-Stanley, G. H.: Simple method for the isolation and purification of total lipids from animal tissues. J. Biol. Chem., 226: 497 (1957).

9. Fujiwara, T. Adams, F. J., Nozaki, M., and Dermer, G. B.: Pulmonary surfactant phospholipids from turkey lung: comparison with rabbit lung. Am. J. Physiol. 218: 218 (1970).

10. Gail, D. B., and Farrell, P. M.: Measurement of phosphatidylcholine precursorscholine, ethanolamine and methionine - in fetal and adult rat lung. Lung, 155 ; 255 (1978).

11. Gluck, L., Kolovich, M. V., Eidelman, A. I., Cordero, L.. and Khazin, A. F. Biochemical development of surface activity in mammalian lung. IV. Pulmonary lecithin synthesis in the human fetus and newborn and etiology of the respiratory distress syndrome. Pediatr. Res., 6: 81 (1972).

12. Goldfine, H.: Lipid chemistry and metabolism. Ann. Rev. Biochem., 37: 303 (1968).

13. Hamburger, V., and Hamilton, H.: A series of normal stages in the development of the chick embryo. J. Morphol., 88: 49 (1951).

14. Lambson, R. O., and Cohn, J. E.: Ultrastructure of the lung of the goose and its lining of surfactant material. Am. J. Anat., 122: 631 (1968).

15. Lowry, O. H., Rosebrough, N. J., Farr, A. L., and Randall, R. J.: Protein measurement with the Folin phenol reagent. J. Biol Chem., 193: 265 (1951).

16. Marin, L., Tordet, C., and Dameron, F. L.: The endocrine control of embryonic lung maturation in the chicken. I. Morphological and biochemical differentiation of lungs after "in ovo" decapitation. Anat. Embryol., 152: 223 (1978).

17. Pattle, R. E.: Lung lining in birds, reptile, and amphibian. Nature (Lond.), 200: 894 (1963).

18. Pattle, R. E.: Lung surfactant and lung lining in birds. In: J. Piiper: Respiratory Function in Birds-Adult and Embryonic. pp. 23-32 (Springer-Verlag, Berlin, 1978).

19. Petrick, P., and Riedel, B.: An osmiophilic bilaminar lining film at the respiratory surfaces of avian lungs. Z. Zellforsch. Mikrosk, Anat., 88: 204 (1968)

20. Sim, M. K and Gwee, M. C. E. A comparative study of the distribution of free choline in the plasma, liver, lung and kidney of the toad, tortoise, chicken, and guinea pig. Comp. Biochem. Physiol., 58C: 37 (1974).

21. Tyler, W. S., and Pangborn, J.: Laminated membrane surface and osmiophilic inclusions in avian lung epithelium. J. Cell Biol., 20: 157 (1964).

22. Weinhold, P. A.: Phospholipid metabolism in the liver and lung of rats during development. Biochem. Biophys. Acta, 106: 540 (1965).

23. Woods, J. E., DeVries, G. W., and Thommes, R. C.: Ontogenesis of the pituitaryadrenal axis in the chick embryo. Gen. Comp. Endocrinol., 17: 407 (1971).

24. The present address of Dr. Suzanne K. Compton is: Department of Physiology and Biophysics, Georgetown University Schools of Medicine and Dentistry, Washington, D. C. 20007.

25. The authors wish to thank Dr. Rodney Ulane for his advice during the course of these experiments.

26. Requests for reprints should be addressed to: Dr. G. C. Goeringer, Department of Anatomy, Georgetown University Schools of Medicine and Dentistry, 3900 Reservoir Road, Washington, D. C. 20007 (USA).

27. This research was supported by N. C. I. Contract NO1-CP-55666, N. I. H. Grants RR 5360 and RR 5306, and a summer research fellowship from the Washington Heart Association to Suzanne K. Compton.

28. Received for publication July 15, 1980.

29. Accepted for publication September 22, 1980. 